\title{
PHARMACOKINETIC MODEL FOR QUANTITATIVE EVALUATION OF THE EPR EFFECT ON ALBUMIN-BASED NANOCARRIERS
}

Ailton A. Sousa-Junior'1, Gustavo C. P. Leite1, Nicholas Zufelato', Francyelli Mello-Andrade², Wanessa C. Pires², Elisângela P. Silveira-Lacerda², Andris F. Bakuzis ${ }^{1}$

${ }^{1}$ Instituto de Física, Universidade Federal de Goiás - Goiânia (GO), Brazil.

2Instituto de Ciências Biológicas, Universidade Federal de Goiás - Goiânia (GO), Brazil.

Understanding the dynamics of the tumor microenvironment is fundamental for the success of new anti-cancer therapies based on tumor targeting drug delivery. It is known that, in an effort to guarantee a continuous flow of oxygen and nutrients, the tumor cells emit molecular signals (VEGF, Vascular Endothelial Growth Factors) to the neighboring endothelial cells, inducing the formation of new blood vessels. It is also known that the endothelium of these new blood vessels shows abnormalities, like intercellular fenestrations, which allow an enhanced permeability and retention of nanostructures within the tumor extracellular matrix - an effect known as EPR (Enhanced Permeability and Retention). However, although this effect is known for over 30 years, very little is known about its kinetics. Therefore, we designed a 4-compartment pharmacokinetic model in order to quantitative evaluate (both analytically and numerically) of the role of the EPR effect on the absorption of multifunctional nanocarriers by tumor tissues. Aliquots $(150 \mathrm{uL})$ of colloidal suspensions containing albumin-based nanocarriers were retro-orbitally administered to a control group (healthy Swiss mice) and to a treatment group (murine-Ehrlich tumor-bearing Swiss mice). Our nanocarriers, named after MalbIR, consisted of manganese ferrite (MnFe2O4) superparamagnetic nanoparticles associated both with molecules of bovine serum albumin (BSA) and with molecules of IR-780 iodide, a near infrared (NIR) fluorescent dye with chemotherapeutic action. The time-evolution of the amount of IR-780 (in pmol) within the retro-orbital, tail and tumor regions was determined via FMT (Fluorescence Molecular Tomography) during the first 8 days after the administration. According to the experimental data, in the first $48 \mathrm{~h}$, the albumin-based nanocarriers accumulate within the tumor region presumably due to: the EPR effect (passive targeting); and to the affinity between BSA (and/or IR-780) molecules and tumor cell membrane receptors (active targeting). The results also enabled the determination of the pharmacokinetic model parameters (first-order intercompartment exchange rate constants, in h-1), consequently bringing a better understanding of the EPR effect kinetics for this type of nanocarrier. Intratumoral accumulation (via EPR effect and presumably via other internalization mechanisms) was clearly observed. The time-evolution profile of the amount of IR-780 in different body regions (retro-orbital sinus, tail and tumor) could be successfully determined by FMT. Albumin-based nanocarriers have long blood half-lives (post-distributive phase), with no toxicity concerns at the assessed concentrations. In particular, our nanocarriers (MalbIR, i.e. MnFe2O4 nanoparticles + BSA + IR-780) show delivery efficiencies (DE) around 15\% of the initial dose (ID): about 5 times higher than the DE measured for the free drug (free IR-780); and about 21 times higher than the median DE value reported by 232 papers over one decade (2005-2015). 